If now $p$ is Fréchet interior to $\mathfrak{S}$ and is a limiting element of $\mathfrak{T}$ a subclass of $\Re$, then, by definition of Fréchet interior, limiting element, and $L^{2}, \mathfrak{S}$ contains an infinity of elements of $\mathfrak{T}$. Therefore $p$ is interior to $\subseteq$ in the sense of $\S 1$. Furthermore if $p$ is interior $(\Re)$ to $\subseteq$ in the sense of $\S 1$, then $\subseteq$ contains an element $q$ (distinct from $\mathfrak{B}$ ) of every subclass $\mathfrak{T}$ of $\mathfrak{R}$ for which $p$ is a limiting element. Then if $p=L_{n} r_{n}$ (distinct) $p$ is a limiting element of the class $\left[r_{n}\right]$. Hence $\mathfrak{S}$ contains $r_{n_{1}}$ distinct from $p$. Since $p$ is a limiting element of the class obtained from $\left[r_{n}\right]$ by removing $r_{n_{1}}\left(L^{2}\right)$ it is evident that at most a finite number of elements of $\left[r_{n}\right]$ are not in $\mathfrak{S}$. Therefore $\left[r_{n}\right]$ is ultimately contained in $\mathfrak{S}$.

T. H. Hildebrandt* has given a definition of interior $(\Re)$ which becomes equivalent to the Fréchet interior $(\Re)$ for systems $\left(\mathfrak{B} ; L^{123}\right)$. This definition omits the condition that the sequence $\left\{r_{n}\right\}$ consist of distinct elements. If then $p=L_{n} r_{n}$ and $r_{n_{0}}$ is repeated infinitely often, in a system $\left(\mathfrak{P} ; L^{123}\right), r_{n_{0}}=p$. That $r_{n_{0}}$ is contained in any class $\mathbb{S}$ to which $p$ is Fréchet interior $(\Re)$ is evident. A restatement of Theorem IV for systems $\left(\mathfrak{P} ; L^{123}\right)$ gives us a generalization of a theorem of Hildebrandt. $\dagger$

Urbana, Ill.

October 28, 1914.

\title{
COMPLETE EXISTENTIAL THEORY OF SHEFFER'S POSTULATES FOR BOOLEAN ALGEBRAS.
}

\author{
BY PROFESSOR L. L. DINES.
}

(Read before the American Mathematical Society, December 30, 1913.)

IN a recent number of the Transactions Sheffer $\ddagger$ presented an elegant and concise set of five postulates for Boolean algebras, and proved them mutually consistent and independent. Professor E. H. Moore§ has suggested a further interesting problem in connection with such sets of postulates, namely the determination of all general implicational relations

* Loc. cit., p. 268 (10).

† Loc. cit., p. 282 (2).

$\ddagger$ H. M. Sheffer, "A set of five postulates for Boolean algebras with application to logical constants," Transactions, vol. 14 (1913), pp. 481-488.

$\S$ E. H. Moore, "Introduction to a form of general analysis," New Haven Mathematical Colloquium, Yale University Press, page 82. 
which exist between properties defined either by the postulates themselves or by the negatives of the postulates. A set of postulates are then said to be completely independent, if and only if no such implicational relations exist. For example, though Sheffer's postulates are independent in the ordinary sense that no four of them imply a fifth, they are not completely independent, for it can be shown that the negative of the first postulate implies the third, fourth, and fifth.

The postulates in question define properties of a system consisting of a class $\Omega$ of undefined elements and an undefined binary rule of combination / between elements of $\Omega$. Any system $\Sigma(\Re, \mid)$ of the prescribed type has, with respect to the five properties defined by the postulates, one of the $2^{5}=32$ characters:

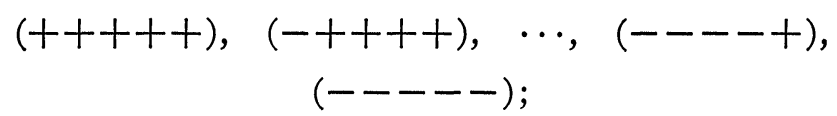

the $i$ th sign of the character being + or - according as $\Sigma$ has or has not the $i$ th property. The body of $2^{5}=32$ propositions stating for the various characters (1) that there does or does not exist a system having the character in question, constitutes what Professor Moore has called "the complete existential theory" of the five postulates.

\section{$\S 1$. Sheffer's Postulates Concerning a System $\Sigma(\Re, \mid)$.}

The five postulates of Sheffer are:

1. There are at least two elements in $\Omega$.

2. Whenever $a$ and $b$ are elements of $\Omega, a \mid b$ is an element of $\Re$.

Definition. $\quad a^{\prime}=a \mid a$.

3. Whenever $a$ and the indicated combinations of $a$ are elements of $\Re$,

$$
\left(a^{\prime}\right)^{\prime}=a .
$$

4. Whenever $a, b$, and the indicated combinations of $a$ and $b$ are elements of $\Re$,

$$
a \mid\left(b \mid b^{\prime}\right)=a^{\prime} .
$$

5. Whenever $a, b, c$, and the indicated combinations of $a, b$, and $c$ are elements of $\Re$,

$$
[a \mid(b \mid c)]^{\prime}=\left(b^{\prime} \mid a\right) \mid\left(c^{\prime} \mid a\right) .
$$


In what follows, the fact that a system $\Sigma$ has the property defined by the $i$ th postulate will be indicated* by placing $i$ as a superscript to $\Sigma$. The fact that $\Sigma$ does not have the property defined by the $i$ th postulate will be denoted by placing $-i$ as a superscript to $\Sigma$. For example we may express the facts that $\Sigma$ satisfies postulates 2 and 3, but does not satisfy postulate 4 by $\Sigma^{23-4}$.

\section{§ 2. Complete Existential Theory.}

THEOREM. For the five postulates 1-5 concerning systems $\Sigma(\Omega, \mid)$, the complete existential theory consists of 14 propositions of non-existence, and 18 propositions of existence. In particular the four postulates 2-5 are completely independent. The non-existences are expressed by the proposition $\dagger$

$$
\Sigma^{-1} \supset \Sigma^{345} \text {. }
$$

To prove proposition (2) we need only note that the hypothesis necessitates either $\Omega^{\text {null }}$ (that is that $\Omega$ have no elements) or $\AA^{\text {singular }}$ (that is that $\AA$ have only one element). In the former case $\Sigma$ satisfies postulates $3,4,5$ vacuously. In the latter case $\Sigma$ satisfies postulates 3, 4, 5 either vacuously or evidently, according as $\Sigma$ does not or does satisfy postulate 2 .

Proposition (2) renders impossible the existence of systems $\Sigma$ with the following 14 characters:

$$
\begin{gathered}
(-+-++),(-++-+),(-+++-),(-++--), \\
(-+-+-),(-+--+),(-+---), \\
(---++),(--+-+),(--++-),(--+--), \\
(---+-),(----+),(-----) .
\end{gathered}
$$

We next give examples of systems $\Sigma$ having each of the other 18 characters as follows: two examples for $\Re^{\text {singular }}$, ten examples for $\Omega^{\text {dual }}$, and six examples for $\Omega^{\text {triple }}$. In each case the class $\Omega$ has the smallest number of elements possible.

* The scheme of notation here described is used by Professor Moore in his General Analysis.

$\dagger$ The symbol $\supset$ used in the statement of this proposition is the symbol of logical implication used by Professor Moore and others. In words, proposition (2) may be stated: "If a system $\Sigma$ does not satisfy postulate 1, then $\Sigma$ does satisfy postulates 3,4 , and $5 . "$ 
Examples for $\Re$ singular.

A class $\Omega$ with single element $m$ furnishes systems $\Sigma$ with the following two characters:

$$
\begin{gathered}
(-++++) \quad \text { when } m / m=m, \\
(--+++) \quad \text { when } m / m \neq m .
\end{gathered}
$$

In the examples for $\Re$ dual, and $\Re$ triple, the operation / will be defined by means of tables. For instance if $\Omega$ has two elements $m$ and $n$; and if $m|m=n, m| n=m, n \mid m=n$, and $n \mid n$ is not an element of $\Omega$, this will be expressed by the table

\begin{tabular}{l|l|l|}
\hline & $m$ & $n$ \\
\hline$m$ & $n$ & $m$ \\
\hline$n$ & $n$ & - \\
\hline
\end{tabular}

Examples for $\Re$ dual.

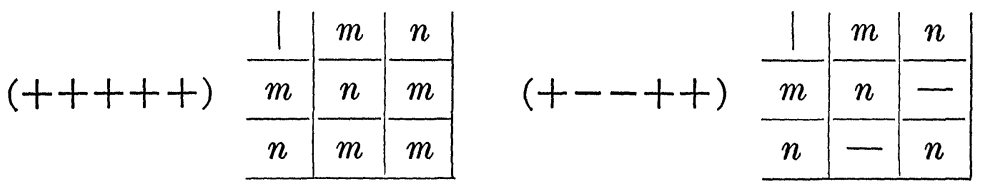

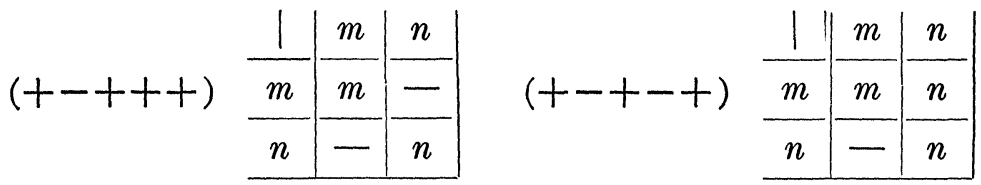

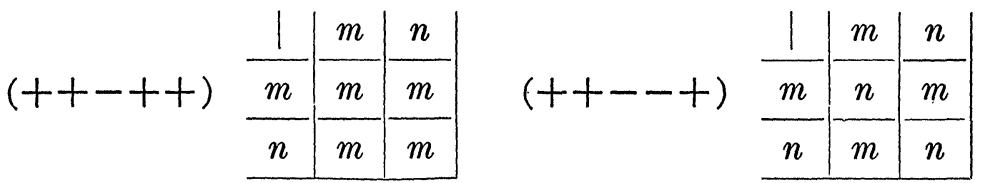

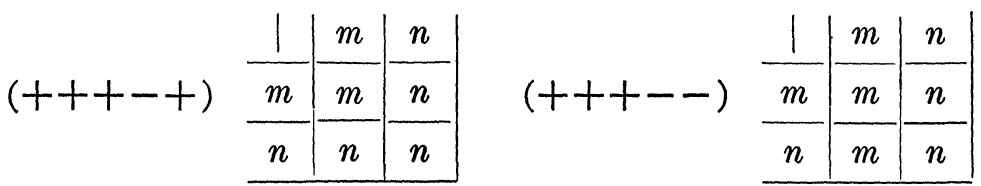




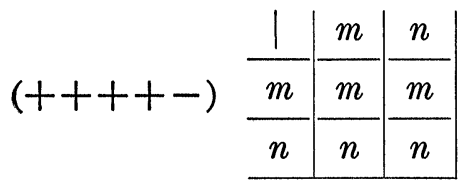

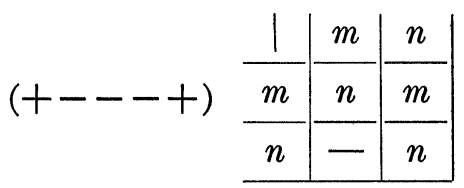

That there are no examples of other characters to be obtained for $\Omega$ dual is shown by the two propositions*

$$
\begin{aligned}
& \AA^{\text {dual }} \cdot \Sigma^{-3}: \supset: \Sigma^{5} \\
& \AA^{\text {dual }} \cdot \Sigma^{-2}: \supset: \Sigma^{5},
\end{aligned}
$$

both of which follow without difficulty from the definitions involved.

Examples for $\Re$ triple.

$$
\begin{aligned}
& (+-++-) \frac{\frac{1}{l}}{\frac{m}{n}}\left|\frac{l}{l}\right| \frac{m}{m}\left|\frac{m}{n}\right| \frac{n}{l} \mid \begin{array}{ll|l|l|l|}
\frac{n}{l} \\
\hline
\end{array}
\end{aligned}
$$

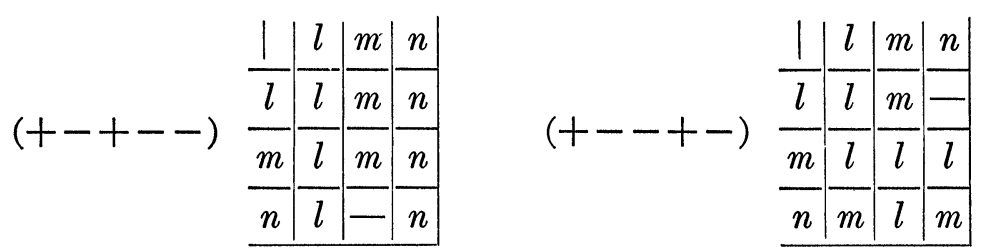

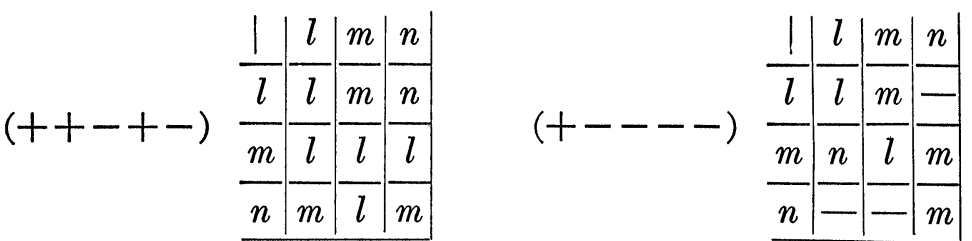

That postulates 2-5 are completely independent follows from the fact that systems have been exhibited having the $2^{4}=16$ characters $(+ \pm \pm \pm \pm)$.

* Proposition (3): If the class $\Re$ has exactly two elements and the system $\Sigma$ does not satisfy postulate 3 , then $\Sigma$ does satisfy postulate 5 .

The interpretation of (4) is similar. 


\section{§3. Concerning a Substitute for Postulate 1.}

The fact that postulate 1 prevents the set of five postulates from being completely independent suggests the desirability of replacing it by another postulate such that the resulting set shall be completely independent. Evidently the new postulate must assume as a minimum number of elements, a greater number than two in order that proposition (2) shall not hold.

If postulate 1 be replaced by the statement that $\Re$ shall have at least three distinct elements, the difficulty is not overcome, for there still exist no systems having the characters

$$
\begin{aligned}
& \text { (-+-+-), (-+---), (--++-), (--+--), } \\
& \text { (-- - - ), (-----), }
\end{aligned}
$$

as is shown by propositions (3) and (4).

It seems rather evident that a completely independent set of postulates could be obtained by postulating as the minimum number of elements of $\Omega$ a sufficiently great number. How great this minimum must be has not however been determined by the author.

UNIVERSITY OF SASKATCHEWAN.

\section{ON THE CHARACTERISTICS OF THE PRINCIPAL MANUALS OF ELEMENTARY GEOMETRY PUBLISHED IN ITALY IN THE COURSE OF THE LAST FIFTY YEARS.*}

BY PROFESSOR MARIO VECCHI.

IN the first half of the nineteenth century, the Elements of Geometry by A. M. Legendre dominated instruction in that subject in Italy. But about 1860, need for a better exposition was felt, and Italian mathematicians, on the initiation of Luigi Cremona, began to turn to the limpid clearness of Euclid. In the year 1867, the Italian government ordered that geometry should be taught on euclidean lines in the classical gymnasia, and in the same year the well-known

* Originally published as Supplement No. I of A. R. Kulischer's translation into Russian of J. W. A. Young's Teaching of Mathematics. The English translation here published has been prepared by A. R. Kulischer. 